DOI: 10.12731/2227-930X-2020-4-18-39

УДК 656

\title{
АНАЛИЗ ПОДХОДОВ К ОЦЕНКЕ РАСПРЕДЕЛЕНИЯ ТРАНСПОРТНОГО СПРОСА МЕЖДУ СПОСОБАМИ ПЕРЕДВИЖЕНИЯ В ГОРОДАХ
}

\section{Литвинов А.В.}

Для поддержки принятия решений при городском транспортном планировании, в том числе при разработке мероприятий по управлению транспортным спросом, необходимо применение эффективных методов оценки распределения транспортного спроса между различными способами передвижения.

Цель исследования - анализ подходов к оценке распределения транспортного спроса между способами передвижения в городах.

Материалы и методы исследования: анализ и систематизация информации о применяемых подходах к оценке распределения транспортного спроса между способами передвижения в городах России и за рубежом (на примере США и Великобритании). Особое внимание уделено источникам, имеющим высокий уровень циттирования и описываюшим подходы, получившие широкое практическое применение.

Результаты: выделены два базовых подхода: прямая оценка на основе данных исследования подвижности населения и оченка на основе использования моделей. Разработана классификация применяемых моделей. Проведенные исследования показали, что современные подходы основываются на применении моделей дискретного выбора. Наиболее перспективными являются модели, описывающие индивидуальное транспортное поведение. На основе проведенного анализа даны рекомендации по развитию подходов к оценке распределения транспортного спроса между способами передвижения и их применению в практике транспортного планирования в российских городах. 
Применение результатов исследования: результаты исследования могут применяться при определении и обосновании подходов к оценке транспортного спроса в рамках городского транспортного планирования.

Ключевые слова: транспортный спрос; исследование подвижности населения; выбор способа передвижения; модель дискретного выбора; мультиномиальный логит; иерархический логит.

\section{ANALYSIS OF APPROACHES TO ESTIMATE THE TRAVEL MODE SHARES IN CITIES}

\section{Litvinov A..}

To support decision-making in urban transport planning including the development of travel demand management measures it is necessary to use efficient methods for estimating the travel mode shares.

The purpose of the research was to analyze approaches to estimate the travel mode shares in cities.

Materials and methods: analysis and systematization of data on the approaches used to estimate the travel mode shares in cities of Russia and abroad (on the example of the USA and Great Britain). Special attention was paid to sources with a high citation index and describing approaches that have received wide practical application.

Results. Two basic approaches were identified: direct estimation based on data from travel surveys and estimation based on the use of models. A classification of the applied models was developed. The analysis showed that modern approaches are based on the use of discrete choice models. The most promising are models that describe individual travel behavior. On the basis of the analysis, recommendations were given for the development of approaches to estimation the travel mode shares and their application in the practice of transport planning in Russian cities.

Practical implications. The results of the research can be used to define and substantiate approaches to estimation the travel mode shares within the framework of urban transport planning. 
Keywords: travel demand; travel survey; travel mode choice; discrete choice model; multinomial logit; hierarchical logit.

\section{Введение}

Оценка и прогнозирование транспортного спроса каждым способом передвижения является важной составляющей процесса планирования транспортной инфраструктуры и сервисов. Транспортный спрос каждым способом передвижения определяется совокупным транспортным спросом и его распределением между способами передвижения.

В современных мультимодальных транспортных системах городов распределение транспортного спроса между способами передвижения является результатом индивидуального выбора, осуществляемого пользователями транспортной системы из множества доступных им альтернатив.

Для поддержки принятия решений при городском транспортном планировании необходимо применение эффективных методов оценки распределения транспортного спроса между различными способами передвижения, которые учитывают закономерности транспортного поведения городского населения. Анализ подходов к оценке и прогнозированию распределения транспортного спроса между способами передвижения в городах является актуальной научно-практической задачей.

\section{Состояние вопроса}

В контексте планирования городских транспортных систем распределение общего транспортного спроса между способами передвижения определяет спрос на инфраструктуру и сервисы каждого способа передвижения. В классической четырехшаговой модели, часто используемой в городском транспортном планировании, оценка распределения транспортного спроса между способами передвижения относится к третьему шагу модели (рисунок 1).

До того, как автомобиль стал массовым средством передвижения, у жителей городов имелись, по сути, две альтернативы - дви- 
жение пешком или на общественном транспорте. Движение пешком осуществлялось, как правило, на небольшие расстояния (до 1-2 км). С увеличением расстояния передвижения всё большую роль играл общественный транспорт.

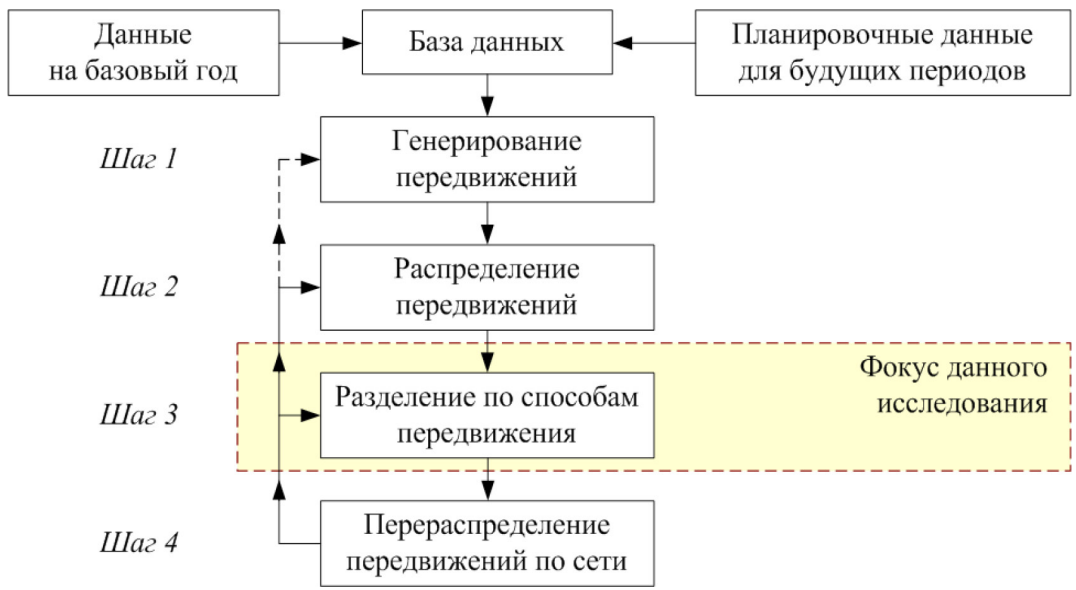

Рис. 1. Классическая четырехшаговая транспортная модель [24]

В условиях массовой автомобилизации населения у большого количества людей появилась еще одна альтернатива в виде личного автомобиля. Задача оценки распределения транспортного спроса между способами передвижения в условиях множественного выбора стала более комплексной.

Увеличение количества автомобилей и активное их использование вызывает ряд негативных последствий, таких как рост транспортных заторов, увеличение затрат на осуществление передвижений людей и доставку товаров, ухудшение экологической ситуации, снижение качества городской среды и т.п.

Дальнейшее экстенсивное наращивание транспортного предложения для удовлетворения всё более возрастающего спроса на передвижения на личных автомобилях связано со значительными социальными и экономическими издержками. Такие негативные последствия могут быть снижены путем разработки и реализа- 
ции мероприятий по управлению транспортным спросом, которые направлены на повышение привлекательности общественного транспорта и движения пешком по сравнению с использованием автомобилей.

В рамках решения данной задачи повышаются требования к методам оценки распределения транспортного спроса между способами передвижения, которые должны быть чувствительными к различными факторам, определяющим транспортное поведение людей.

Целью исследования является анализ подходов к оценке распределения транспортного спроса между способами передвижения в городах.

Задачи исследования:

- обзор существующих подходов к оценке распределения транспортного спроса между способами передвижения в городах и анализ практики их применения в России и за рубежом;

- разработка рекомендаций по развитию подходов к оценке распределения транспортного спроса между способами передвижения и их применению в российских городах.

\section{Материалы и методы}

Для выполнения обзора рассмотрены источники информации на русском и английском языках. Зарубежный опыт описан на примере США и Великобритании. Обзор ранее выполненных работ представлен в хронологическом порядке. Особое внимание уделено работам, имеющим высокий уровень цитирования и описывающим подходы к оценке распределения транспортного спроса, получившие широкое практическое применение. При проведении обзора применяемых методов и моделей учитывается также контекст тех задач транспортного планирования, для которых эти методы и модели были разработаны и использованы.

Обозначения в формулах, используемых в обзоре, могут отличаться от обозначений, используемых в первоисточниках. Одни и те же переменные во всех формулах для лучшего восприятия обо- 
значены одинаково. Индекс «М» соответствует моторизированным способам передвижения (с использованием транспорта), индекс «Х» - общественному транспорту; индекс «С»- индивидуальному транспорту (автомобилю), индекс «W» - способу передвижения пешком.

На основе проведенного обзора выполнен совместный анализ используемых подходов, включая классификационный анализ применяемых моделей.

\section{Результаты и обсуждение}

Подходы к оценке распределения транспортного спроса между способами передвижения в российской практике транспортного планирования

В послевоенные годы (после 1945 года) для оценки распределения транспортного спроса между способами передвижения широкое распространение получил коэффициент пользования транспортом, отражающий долю передвижений с использованием транспорта в общем объеме передвижений всеми способами [1, 7, 10 и др.].

Для практических расчетов широкое применение получила формула расчета коэффициента пользования транспортом в зависимости от расстояния передвижения, предложенная Г.В. Шелейховским [11]:

$$
\varphi_{i j, M}=\lg \frac{l_{i j}}{l_{0}} / \lg \frac{l_{0}}{l_{1}},
$$

где $l_{i j}$ - расстояние передвижения между транспортными зонами $i$ и $j ; 1 \mathrm{~g}$ - десятичный логарифм; $l_{0}$ - нижнее пороговое значение расстояния, ниже которого практически все передвижения осуществляются пешком (около 0,5 км); $l_{1}$ - верхнее пороговое значение расстояния, выше которого практически все передвижения осуществляются с использованием транспорта (около 2 км).

Доля передвижений пешком при этом определялась как $\varphi_{i j, W}=1-\varphi_{i j, M}$.

А.А. Поляков предложил определять коэффициент пользования транспортом с учетом цели передвижения [7]. М.С. Фишельсон 
отмечал, что на значение коэффициента пользования транспортом помимо расстояния передвижения оказывают влияние другие факторы, включая скорость сообщения, удобство поездки и т.п. [10].

По мере роста автомобилизации населения возникла необходимость при расчетах транспортного спроса учитывать также спрос на индивидуальный транспорт. Общий коэффициент пользования транспортом был разделен на коэффициент пользования массовым общественным транспортом и коэффициент пользования индивидуальным транспортом [6]:

$$
\varphi_{i j, X}=\varphi_{i j, M} \cdot \psi_{i j, X} ; \varphi_{i j, C}=\varphi_{i j, M} \cdot \psi_{i j, C},
$$

где $\psi_{i j, X}$ и $\psi_{i j, C}-$ доли транспортных передвижений с использованием массового общественного и индивидуального транспорта соответственно.

Ю.С. Кирзнер для условий города Ленинграда построил регрессионные зависимости для определения доли транспортных передвижений из дома на работу, осуществляемых владельцами автомобилей, с использованием массового общественного транспорта для зимнего и летнего периода [2]:

$$
\psi_{i j, X}=a \cdot \exp \left(b \cdot t_{i j, X} / t_{i j, C}\right),
$$

где $a, b$ - коэффициенты регрессии; $t_{i j, X}$ и $t_{i j, C}-$ время передвижения между транспортными зонами $i$ и $j$ на общественном и индивидуальном транспорте соответственно; ехр - экспоненциальная функция.

Доля транспортных передвижений с использованием индивидуального транспорта определялась при этом как: $\psi_{i j, C}=1-\psi_{i j, X}$.

Модель распределения транспортного спроса между способами передвижения (формулы 2 и 3 ) являлась частью итеративной процедуры комплексной транспортной модели [6] (см. также рисунок 1).

Оценка параметров регрессионных моделей осуществлялась на основе данных социологических исследований подвижности населения, в рамках которых собиралась информация о характеристиках передвижений, совершенных респондентом за определенный период (как правило, сутки). 
Социологические исследования подвижности населения использовались не только для построения моделей, но и для прямой оценки существующего распределения транспортного спроса между способами передвижения. Описание подходов к проведению исследований представлено в работах $[1,8]$. Большой объем исследований подвижности населения в городах был проведен в рамках Всесоюзной переписи населения в 1970 году.

Описанный выше подход к оценке распределения транспортного спроса между способами передвижения (формулы 2 и 3) базируется на соотношении времени передвижения на общественном и индивидуальном транспорте. Помимо времени передвижения среди факторов, влияющих на выбор между индивидуальным и общественным транспортом, О.К. Кудрявцев выделяет удобство и комфорт поездки, близость расположения стоянки для автомобиля, наличие внеуличного транспорта [3].

В работе [4] А.С. Михайлов в качестве критерия выбора населением способа передвижения определил критерий, включающий временные и стоимостные затраты на передвижение, а также душевой доход лица, осуществляющего передвижение.

Ввиду ряда существенных ограничений модели О.К. Кудрявцева и А.С. Михайлова не получили широкого практического применения.

В современной российской практике транспортного планирования для оценки распределения транспортного спроса между способами передвижения часто используется модель мультиномиального логита [9]:

$$
P_{i j m}=\frac{e^{-\lambda c_{i j m}}}{\sum_{n} e^{-\lambda c_{i j n}}} .
$$

где $P_{i j m}$ - доля передвижений способом $m$ между транспортными зонами $i$ и $j ; c_{i j m}$ - обобщенные затраты на передвижение, включающие время, плату за проезд и т.д.; $\lambda$ - параметр модели.

Данная модель (формула 4) использовалась в рамках комплексной транспортной модели Московского региона [9]. Такая модель относится к моделям дискретного выбора. Более подробно модели 
дискретного выбора описаны в разделе, относящемуся к обзору зарубежного опыта.

Подходы коценке распределения транспортного спроса между способами передвижения в зарубежной практике транспортного планирования

Обзор моделей транспортного спроса, разработанных до середины 70-х годов XX века, представлен в работе T.A. Domencich и D. McFadden [19].

В 50-60 годы XX века оценка распределения транспортного спроса между способами передвижения осуществлялась на основе регрессионных моделей вида:

$$
\frac{N_{i j, C}}{N_{i j, X}}=f\left(t_{i j, C}, t_{i j, X}, S E_{i}, L U_{j}\right),
$$

где $N_{i j, C}$ и $N_{i j, X}$ - количество передвижений между зонами $i$ и $j$ на индивидуальном и общественном транспорте; $S E_{i}-$ социальноэкономические характеристики зоны $i ; L U_{j}$ - характеристики землепользования в зоне $j$.

В ранних моделях транспортного спроса шаг распределения транспортного спроса между способами передвижения осуществлялся сразу за шагом генерирования передвижений на основе данных о социально-экономических характеристиках населения [24]. Как правило, в моделях рассматривались только два способа передвижения: общественный и индивидуальный транспорт [19].

Модели оценки распределения транспортного спроса между способами передвижения разрабатывались не только в разрезе корреспонденций между транспортными зонами $i j$, а также для города в целом на основе данных о характеристиках города, таких как уровень автомобилизации, плотность населения и т.п. Пример такой модели для городов США представлен в работе [14] со ссылкой на результаты американских исследований середины XX века:

$$
\varphi_{C}=f\left(n_{a} ; \rho_{\text {pop }}\right),
$$

где $\varphi_{C}-$ доля передвижений на автомобиле;

$$
\begin{aligned}
& h_{a}^{c} \text { - количество семей, приходящихся на один автомобиль; } \\
& \rho_{\text {рор }} \text { - плотность населения города, чел./км². }
\end{aligned}
$$


В США в 60-70-е годы XX века были разработаны модели дискретного выбора. В отличии от регрессионной модели (формула 5) рассматривается не доля передвижений между транспортными зонами каждым способом, а вероятность выбора способа передвижения лицом, принимающим решение.

Основной постулат моделей дискретного выбора в приложении к выбору способа передвижения: вероятность выбора отдельными лицами данного способа передвижения есть функция их социально-экономических характеристик и относительной привлекательности данного способа передвижения [24].

В основе моделей дискретного выбора лежит теория случайной полезности $[19,26]$. Разработка теории случайной полезности способствовала более глубокому пониманию механизмов принятия решений при осуществлении передвижений. Комплексное описание теории случайной полезности и моделей семейства логит представлено в работах T.A. Domencich и D. McFadden [19] и M. Ben-Akiva и S.R. Lerman [12].

Полезность для пользователя $q$ способа передвижения $m[12,19]$ :

$$
U_{m q}=V_{m q}+\varepsilon_{m q}
$$

где $V_{m q}$-измеряемая или систематическая составляющая полезности способа передвижения $m$ для пользователя $q$, которая является функцией характеристик, измеренных и учитываемых в модели; $\varepsilon_{m q}-$ случайная составляющая, отражающая особые вкусы лица $q$, осуществляющего выбор, а также ошибки измерения или наблюдения, допущенные при разработке модели.

Наиболее простой и распространенной функциональной формой модели дискретного выбора является модель мультиномиального логита [19]:

$$
P_{m q}=\frac{e^{V_{m q}}}{\sum_{n} e^{V_{n q}}},
$$

где $V_{m q}$ - систематическая составляющая полезности способа передвижения $m$ для лица $q$, осуществляющего выбор.

Данная модель базируется на допущении, что случайная составляющая полезности $\varepsilon_{m q}$ имеет распределение Гумбеля [19]. 
Наиболее простая форма функции систематической полезности [24]:

$$
V_{m q}=\sum \beta_{k m} X_{m k q}
$$

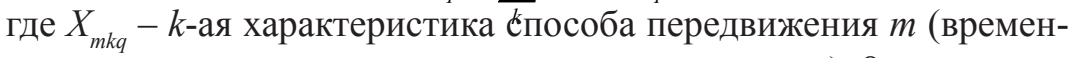
ные и стоимостные затраты на передвижение и т.п.); $\beta_{k m}$ - параметры модели, оцениваемые на основе данных.

Модель мультиномиального логита имеет ограничение, связанное со свойством независимости от нерелевантных альтернатив (IID). Вместо модели мультиномиального логита может быть применена модель иерархического логита [24].

Иерархическая модель применительно к задаче выбора способа передвижения предполагает разделение способов передвижения на несколько непересекающихся множеств, называемых гнездами. Способы передвижения, имеющие одинаковую корреляцию случайных составляющих полезности, располагают в одном гнезде, а без корреляции - в разных гнездах. Фундаментальные разработки модели иерархического логита представлены в работах A.J. Daly и S. Zachary [18], H.C.W.L. Williams [26], D. McFadden [22].

Для модели иерархического логита с одним уровнем вложенности вероятность выбора пользователем $q$ способа передвижения $m$ может быть представлена в виде [25]:

$$
P_{m q}=\frac{e^{V_{m q} / \lambda_{n}}\left(\sum_{j \in n} e^{V_{j q} / \lambda_{n}}\right)^{\lambda_{n}-1}}{\sum_{l=1}^{N}\left(\sum_{j \in l} e^{V_{j q} / \lambda_{l}}\right)^{\lambda_{l}} .}
$$

где $\lambda_{n}$ - параметр, определяющий степень независимости случайных составляющих полезности среди способов передвижения в гнезде $n ; N$ - количество гнезд.

В работе [22] D. McFadden рассмотрел модель иерархического логита как частный случай семейства моделей дискретного выбора с распределением случайной составляющей полезности в соответствии с обобщенным распределением экстремальных значений (GEV).

Использование вместо распределения Гумбеля нормального распределение случайной составляющей полезности $\varepsilon_{m q}$ привело к созданию модели мультиномиального пробита. Анализ пробит мо- 
делей представлен в работах C.F. Daganzo [17], J.L. Horowitz [20], M. Ben-Akiva и S.R. Lerman [12].

В конце 90-х годов XX века появились модели, позволяющие учитывать ненаблюдаемую неоднородность между индивидуумами и / или взаимодействия между ненаблюдаемыми составляющими функций полезности различных альтернатив. Среди таких моделей можно выделить модель смешанного логита, разработанную D. McFadden и K. Train [23], M. Ben-Akiva и D. Bolduc [13].

$\mathrm{B}$ практике транспортного планирования среди моделей дискретного выбора наиболее широкое распространение получили модели мультиномиального и иерархического логита. Пример применения модели мультиномиального логита для моделирования выбора способа передвижения в Лондоне в рамках комплексной транспортной модели представлен в работе П.У. Бонсалла [7]. Множество других примеров представлено в работах $[16,24]$.

J. Ortúzar [24] отмечает, что оценка параметров моделей дискретного выбора, как правило, не может быть выполнена с использованием стандартных методов аппроксимации, таких как метод наименьших квадратов, поскольку зависимая переменная модели есть ненаблюдаемая вероятность (между 0 и 1 ), а для каждого наблюдения имеет место индивидуальный выбор (0 или 1). Для оценки параметров модели выбора способа передвижения предложен метод максимизации функции вероятности [24].

Основными источниками данных для разработки моделей являются результаты социологический исследований. Традиционные подходы основываются на исследовании транспортного поведения в реальных условиях - исследовании выявленных предпочтений. Данные исследования дают информацию о респонденте, совершенных им передвижениях, включая информацию о фактическом выборе способа передвижения [24]. Альтернативным подходом является исследование гипотетического транспортного поведения исследование заявленных предпочтений, которое концептуально эквивалентно лабораторному эксперименту. Обзор теоретических и практических аспектов исследований заявленных предпочтений 
представлен в работах J.J. Louviere [21], J. Ortúzar [24]. Подходы к оценке параметров моделей на основе объединенных данных исследований выявленных и заявленных предпочтений представлены в работах $[14,15]$. В работе [25] представлены подходы к оценке параметров моделей на основе панельных данных.

Анализ подходов к оценке распределения транспортного спроса между способами передвижения в городах

Краткое резюме результатов обзора источников представлено в таблице 1.

Таблица 1.

Краткое резюме результатов обзора источников

\begin{tabular}{|c|c|c|}
\hline $\begin{array}{c}\text { Пе- } \\
\text { риод } \\
\text { (годы) }\end{array}$ & Русскоязычные источники & Англоязычные источники \\
\hline 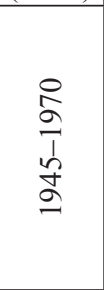 & $\begin{array}{l}\text { - Модель оценки коэффи- } \\
\text { циента пользования транс- } \\
\text { портом (Г.В. Шелейхов- } \\
\text { ский, А.А. Поляков и др.). } \\
\text { - Методические подходы } \\
\text { к исследованию внутри- } \\
\text { городской подвижности } \\
\text { населения. }\end{array}$ & $\begin{array}{l}\text { - Регрессионные модели распределения } \\
\text { спроса между индивидуальным и обще- } \\
\text { ственным транспортом (M.J. Fertal и др.). } \\
\text { - Модели бинарного выбора (S.L. Warner, } \\
\text { R.G. McGillivray и др.). } \\
\text { - Метод оценки моделей на основе выяв- } \\
\text { ленных предпочтений. }\end{array}$ \\
\hline 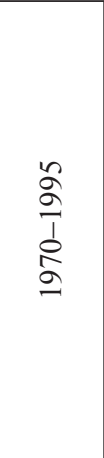 & $\begin{array}{l}\text { - Регрессионные моде- } \\
\text { ли оценки доли передви- } \\
\text { жений на обществен- } \\
\text { ном транспорте в общем } \\
\text { объеме транспортных } \\
\text { передвижений (М.С. Фи- } \\
\text { шельсон, Ю.С. Кирзнер, } \\
\text { О.К. Кудрявцев и др.). }\end{array}$ & $\begin{array}{l}\text { - Модели мультиномиального логита } \\
\text { (D. McFadden, M. Ben-Akiva и др.). } \\
\text { - Модели иерархическо- } \\
\text { го логита (A.J. Daly, S.D. Zachary, } \\
\text { H.C.W.L. Williams, D. McFadden, M. Ben- } \\
\text { Akiva и др.) и другие модели семейства } \\
\text { GEV (D. McFadden и др.). } \\
\text { - Модели мультиномиального пробита } \\
\text { (C.F. Daganzo, J.L. Ноrowitz, M. Ben- } \\
\text { Akiva и др.). } \\
\text { - Метод оценки моделей на основе дан- } \\
\text { ных заявленных предпочтений } \\
\text { (J.J. Louviere, J. Ortúzar и т.д.). }\end{array}$ \\
\hline $\begin{array}{l}\text { đิ } \\
\text { ஸे } \\
\stackrel{2}{\alpha}\end{array}$ & \begin{tabular}{|l} 
- Модель вероятности \\
выбора способа передви- \\
жения на основе критерия \\
транзакционных издержек \\
(А.С. Михайлов).
\end{tabular} & $\begin{array}{l}\text { - Модели смешанного логита } \\
\text { (D. McFadden, K. Train, M. Ben-Akiva, } \\
\text { D. Bolduc и др.). } \\
\text { - Метод оценки моделей смешанного } \\
\text { логита на основе панельных данных } \\
\text { (K. Train и др.). }\end{array}$ \\
\hline
\end{tabular}


На основе проведенного анализа можно выделить следующие подходы, используемые для оценки распределения транспортного спроса между способами передвижения в городах:

- подход, основанный на прямой оценке на основе данных исследования подвижности населения;

- подход, основанный на использовании моделей.

Подход, основанный на прямой оценке, позволяет оценить существующее распределение транспортного спроса между способами передвижения в городах. Данный подход не пригоден для прогнозирования распределения транспортного спроса, но при этом обеспечивает необходимые данные для построения прогнозных моделей.

Признак классификации

Уровень сложности модели

Интегрированность в комплексную модель

Уровень агрегирования

Учет случайных факторов

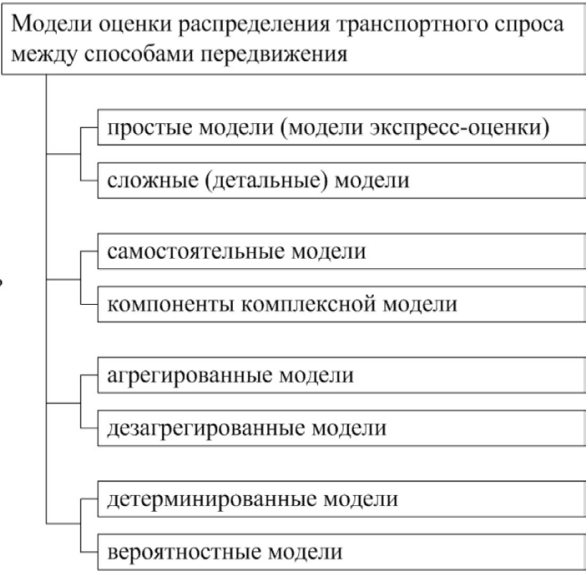

Рис. 2. Классификация моделей оценки распределения транспортного спроса между способами передвижения

Среди моделей в зависимости от уровня детализации представления спроса и / или факторов, которые на него влияют, можно выделить агрегированные и дезагрегированные модели (рисунок 2). В агрегированных моделях включенные в модель переменные относятся к группе пользователей (например, среднее время всех передвижений между двумя транспортными зонами, среднее количество автомобилей, приходящихся на домохозяйство, и т.п.). В 
дезагрегированных моделях переменные относятся к отдельному пользователю (например, время передвижения между фактическими пунктами отправления и назначения, количество автомобилей в домохозяйстве конкретного индивида и т.п.). Модели дискретного выбора могут применяться в качестве функциональной формы как агрегированных (формула 4), так и дезагрегированных моделей (формула 8).

Поскольку распределение транспортного спроса между способами передвижения формируется в результате индивидуального выбора, дезагрегированные модели, как правило, лучше описывают индивидуальное транспортное поведение. Для оценки распределения транспортного спроса на основе дезагрегированных моделей выбора способа передвижения используется процедура агрегирования. В российской практике транспортного планирования не удалось найти примеров разработки и применения дезагрегированных моделей, в то время как за рубежом такие модели получили широкое распространение.

В зависимости от учета в моделях случайных факторов можно выделить вероятностные и детерминированные модели. Более ранние модели (формулы 3 и 5) относятся к детерминированным моделям. Модели, основанные на теории дискретного выбора, являются вероятностными моделями (формулы 8 и 10). Поскольку выбор способа передвижения имеет случайный характер, вероятностные модели, как правило, являются предпочтительными.

Модели распределения транспортного спроса между способами передвижения могут быть как самостоятельными моделями, так и частью комплексных моделей транспортного спроса (рисунок 1). Включение модели распределения транспортного спроса между способами передвижения в комплексную транспортную модель позволяет учитывает множество обратных связей, оказывающих влияние на формирование транспортного спроса. Если такое влияние отсутствует или им можно пренебречь, то модель оценки распределения транспортного спроса между способами передвижения может использоваться в качестве самостоятельной модели. 
Как для прямой оценки распределения транспортного спроса между способами передвижения, так и для оценки с использованием моделей необходимо проведение соответствующих исследований транспортного спроса. За последние 30 лет систематических исследований подвижности населения в российских городах практически не проводилось. Крайне мало публикаций по полученным результатам исследований, а также разработанным на их основе моделям.

Оценка распределения транспортного спроса с использованием большинства из вышеописанных моделей осуществляется на детальном уровне с учетом характеристик отдельных пользователей или групп населения, характеристик качества обслуживания каждым способом передвижения. Помимо таких детальных моделей могут применяться модели для экспресс-оценки распределения транспортного спроса на основе нескольких ключевых характеристик города, влияющих на формирование структуры транспортного спроса. К таким характеристикам могут относиться, например, численность населения, уровень его автомобилизации и другие, данные для определения которых доступны на основе статистического учета. Пример модели для экспресс-оценки доли передвижений на автомобилях в городах США представлен выше (формула 6).

\section{Выводы}

По результатам проведенного анализа подходов к оценке распределения транспортного спроса между способами передвижения сделаны следующие выводы и рекомендации:

- Оценка распределения транспортного спроса может быть осуществлена напрямую на основе данных исследования подвижности населения или на основе применения моделей. Модели могут быть использованы для исследования транспортного поведения населения и прогнозирования изменений распределения транспортного спроса между способами передвижения при разработке мероприятий по развитию городских транспортных систем (что будет, если ...). 
- Современные подходы к оценке распределения транспортного спроса между способами передвижения основываются на применении моделей дискретного выбора.

- В западных странах широкое применение получили дезагрегированные модели, рассматривающие транспортный спрос на индивидуальном уровне. Такие модели лучше описывают транспортное поведение и поэтому являются более перспективными. В России дезагрегированные модели транспортного спроса в практике транспортного планирования не используются.

- В России крайне скудная эмпирическая база исследований транспортного спроса и поведения, что сдерживает развитие научно-методической базы для транспортного моделирования и планирования. Для увеличения объема исследований может быть реализована программа исследования подвижности населения на национальном (федеральном) уровне. Такие программы широко применяются в большинстве западных стран.

- Существует потребность в моделях для экспресс-оценки распределения транспортного спроса между способами передвижения, в особенности, доли передвижения на личных автомобилях. Такие модели могут быть полезным инструментом при проведении предварительного анализа транспортной ситуации в городах (в особенности в условиях отсутствия данных исследований подвижности населения), а также при стратегическом транспортном планировании.

- Применяемые в зарубежных странах подходы к оценке и прогнозированию транспортного спроса могут быть адаптированы и применены в российских городах с учетом локальных демографических, социально-экономических и градостроительных условий.

\section{Список литературы}

1. Ваксман С.А. Социально-экономические проблемы прогнозирования систем массового пассажирского транспорта в городах. Екатеринбург: изд-во Урал. гос. экон. ун-та, 1996. 289 с. 
2. Кирзнер Ю.С. Прогнозирование развития индивидуального транспорта в городе. Методологический аспект // Городской транспорт и организация городского движения: тезисы докладов к научно-технической конференции. Свердловск. 1973. С. 176-181.

3. Кудрявцев О.К., Федутинов Ю.А., Чуверин И.И. Транспорт городских центров. М.: Транспорт, 1978. 110 с.

4. Михайлов А.С. Управление рынком перемещений городского населения. Алматы: НИЦ Гылым, 2003. 237 с.

5. Моделирование пассажиропотоков в транспортной системе: (Оценка вариантов развития транспортной системы и анализ чувствительности модели) / П.У. Бонсалл, А.Ф. Чемперноун, А.К. Мейсон, А.Г. Уилсон. М.: Транспорт, 1982. 207 с.

6. Овечников Е.В., Фишельсон М.С. Городской транспорт. М.: Высшая школа, $1976.352 \mathrm{c}$.

7. Поляков А.А. Организация движения на улицах и дорогах. М.: Транспорт, 1965. $376 \mathrm{c.}$

8. Руководство по проведению транспортных обследований в городах. М.: Стройиздат, 1982. 72 с.

9. Транспортная модель Московского региона / А.Э. Воробьёв, А.Ю. Титов, В.А. Гаврилин, А.Ю. Меньшутин, И.А. Бахирев // Вычислительные технологии в естественных науках. Методы суперкомпьютерного моделирования: Сборник трудов. Сер. «Механика, управление и информатика». Москва. 2015. С. 49-62.

10. Фишельсон М.С. Транспортная планировка городов. М.: Высш. шк., 1985. 239 с.

11. Шелейховский Г.В. Композиция городского плана как проблема транспорта. М.: Гипрогор, 1946. 129 с.

12. Ben-Akiva M., Lerman S.R. Discrete choice analysis: theory and application to travel demand. $1985.390 \mathrm{p}$.

13. Ben-Akiva M., Bolduc D. Multinomial probit with a logit kernel and a general parametric specification of the covariance structure. Working Paper, Departament d'Economique, Universite Laval, 1996.

14. Ben-Akiva M., Morikawa T. Estimation of switching models from revealed preferences and stated intentions. Transportation research A, no.24, 1990. pp. 149-164. 
15. Bradley M., Daly A.J. Estimation of logit choice models using mixed stated preference and revealed preference information. In Proceeding of the $20^{\text {th }}$ PTRC summer annal meeting, University of Manchester, England, 1992.

16. Cascetta E. Transportation Systems Engineering: Theory and Methods. Kluwer Academic Publishers, 2011. 742 p.

17. Daganzo C.F. Multinomial probit: The theory and its application to demand forecasting. Academic Press, New York, 1979.

18. Daly A.J., Zachary S. Improved multiple choice models. In D.A. Hensher and M.Q. Dalvi, Determinants of travel choice. Saxon House, Westmead, 1978.

19. Domencich T.A., McFadden D. Urban travel demand. A behavioral analysis. North-Holland Publishing Company, 1975. 215 p.

20. Horowitz J.L. Specification tests for probabilistic choice models. Transportation research A, no. 16, 1982, pp. 383-394.

21. Louviere J.J., Hensher D.A, Swait J.D. Stated choice methods: analysis and application. Cambridge University Press, Cambridge, 2000.

22. McFadden D. Econometric models of probabilistic choice. In C. Manski and D. McFadden (eds.), Structural analysis of discrete data with econometric applications. The MIT Press, Cambridge, Mass., 1981.

23. McFadden D., Train K. Mixed MNL models for discrete response. Journal of Applied Econometrics, no. 15, 2000, pp. 447-470.

24. Ortúzar J. de L., Willumsen L.G. Modelling Transport. John Wiley \& Sons Ltd., 2011. 586 p.

25. Train K.E. Discrete choice methods with simulation. Cambridge University Press, 2009. 388 p.

26. Williams H.C.W.L. On the formation of travel demand models and economic evaluation measures of user benefit. Environment and Planning A, no. 9, 1977, pp. 285-344.

\section{References}

1. Vaksman S.A. Social'no-jekonomicheskie problemy prognozirovanija sistem massovogo passazhirskogo transporta v gorodah [Socio-economic problems of mass passenger transport systems forecasting in cities]. Ekaterinburg, 1996. 289 p. 
2. Kirzner Ju.S. Prognozirovanie razvitija individual'nogo transporta $\mathrm{v}$ gorode. Metodologicheskij aspect [Individual transport development forecasting in a city. Methodological aspect]. Gorodskoj transport i organizacija gorodskogo dvizhenija: tezisy dokladov k nauchno-tehnicheskoj konferencii [Urban transport and organization of urban traffic: abstracts for the scientific and technical conference]. Sverdlovsk, 1973, pp. 176-181.

3. Kudrjavcev O.K., Fedutinov Ju.A., Chuverin I.I. Transport gorodskih centrov [Transport of urban centers]. Moskva.: Transport publ., 1978. 110 p.

4. Mihajlov A.S. Upravlenie rynkom peremeshhenij gorodskogo naselenija [Managing the urban travel market]. Almaty: NIC Gylym publ., 2003. 237 p.

5. Bonsall P.W., Chempernoun A.F., Mason A.C., Wilson A.G. Modelirovanie passazhiropotokov $v$ transportnoj sisteme: (Ocenka variantov razvitija transportnoj sistemy i analiz chuvstvitel'nosti modeli) [Transport modelling: Sensitivity analysis and policy testing]. Moskva: Transport Publ., 1982. 207 p.

6. Ovechnikov E.V., Fishel'son M.S. Gorodskoj transport [Urban transport]. Moskva: Vysshaja shkola publ., 1976. 352 p.

7. Poljakov A.A. Organizacija dvizhenija na ulicah $i$ dorogah [Organization of traffic on streets and roads]. Moskva: Transport publ., 1965. 376 p.

8. Rukovodstvo po provedeniju transportnyh obsledovanij v gorodah [Guide to conducting urban transport surveys]. Moskva: Strojizdat publ., 1982. $72 \mathrm{p}$.

9. Vorob'jov A.Je., Titov A.Ju., Gavrilin V.A., Men'shutin A.Ju., Bahirev I.A. Transportnaja model' Moskovskogo regiona [Moscow region transport model]. Vychislitel'nye tehnologii v estestvennyh naukah. Metody superkomp'juternogo modelirovanija: Sbornik trudov. Ser. «Mehanika, upravlenie i informatika» [Computational technologies in natural sciences. Supercomputer modeling methods: Proceedings. Ser. "Mechanics, Management and Informatics"]. Moscow, 2015, pp. 49-62.

10. Fishel'son M.S. Transportnaja planirovka gorodov [Urban transport planning]. Moskva: Vysshaja shkola publ., 1985. 239 p.

11. Shelejhovskij G.V. Kompozicija gorodskogo plana kak problema transporta [City plan composition as a transport problem]. Moscow: Giprogor publ., 1946. 129 p. 
12. Ben-Akiva M., Lerman S.R. Discrete choice analysis: theory and application to travel demand, 1985. $390 \mathrm{p}$.

13. Ben-Akiva M., Bolduc D. Multinomial probit with a logit kernel and a general parametric specification of the covariance structure. Working Paper, Departament d'Economique, Universite Laval, 1996.

14. Ben-Akiva M., Morikawa T. Estimation of switching models from revealed preferences and stated intentions. Transportation research $A$, no.24, 1990, pp. 149-164.

15. Bradley M., Daly A.J. Estimation of logit choice models using mixed stated preference and revealed preference information. In Proceeding of the $20^{\text {th }}$ PTRC summer annal meeting, University of Manchester, England, 1992.

16. Cascetta E. Transportation Systems Engineering: Theory and Methods. Kluwer Academic Publishers, 2011. 742 p.

17. Daganzo C.F. Multinomial probit: The theory and its application to demand forecasting. Academic Press, New York, 1979.

18. Daly A.J., Zachary S. Improved multiple choice models. In D.A. Hensher and M.Q. Dalvi, Determinants of travel choice. Saxon House, Westmead, 1978.

19. Domencich T.A., McFadden D. Urban travel demand. A behavioral analysis. North-Holland Publishing Company, 1975. 215 p.

20. Horowitz J.L. Specification tests for probabilistic choice models. Transportation research A, no. 16, 1982, pp. 383-394.

21.Louviere J.J., Hensher D.A, Swait J.D. Stated choice methods: analysis and application. Cambridge University Press, Cambridge, 2000.

22. McFadden D. Econometric models of probabilistic choice. In C. Manski and D. McFadden (eds.), Structural analysis of discrete data with econometric applications. The MIT Press, Cambridge, Mass., 1981.

23. McFadden D., Train K. Mixed MNL models for discrete response. Journal of Applied Econometrics, no. 15, 2000, pp. 447-470.

24. Ortúzar J. de L., Willumsen L.G. Modelling Transport. John Wiley \& Sons Ltd., 2011. 586 p.

25. Train K.E. Discrete choice methods with simulation. Cambridge University Press, 2009. 388 p. 
26. Williams H.C.W.L. On the formation of travel demand models and economic evaluation measures of user benefit. Environment and Planning $A$, no. 9, 1977, pp. 285-344.

\section{ДАННЫЕ ОБ АВТОРЕ}

Литвинов Александр Владимирович, аспирант

ОАО «НИИАТ»

125480, Россия, г. Москва, ул. Героев Панфиловиев, 24

alitvinov85@gmail.com

\section{DATA ABOUT THE AUTHOR}

Litvinov Alexander Vladimirovich, $\mathrm{PhD}$ student

JSC NIIAT

24, Geroyev Panfilovtsev Str., Moscow, 125480, Russian Federation

alitvinov85@gmail.com 